\title{
Utility of 6-Amino-2-thiouracil as a Precursor for the Synthesis of Pyrido[2,3-d]Pyrimidines and their in vitro and in vivo
} Biological Evaluation.

\author{
Nadia Ragab Mohamed, ${ }^{* 1}$ Manal M. T. El-Saidi, ${ }^{1}$ \\ Yasser Mahmoud Ali, ${ }^{1}$ Mohamed Hilmy Elnagdi. ${ }^{2}$ \\ ${ }^{1}$ Department of Photochemistry, National Research Center, \\ Dokki, Giza, Egypt. \\ ${ }^{2}$ Department of Chemistry, Faculty of Science, Cairo University, \\ Giza, Egypt.
}

\section{Abstract}

The condensation of 6-amino-2-thiouracil 1 with aromatic aldehydes afforded azamethine derivatives $\mathbf{3 a , b}$. The formed azamethines underwent [4+2] cycloaddition with enaminones $4 \mathrm{a}-\mathrm{c}$ and enaminonitrile 9 to form the corresponding condensed pyrimidines $\mathbf{8 a}-\mathbf{f}$ and $\mathbf{1 1 a}, \mathbf{b}$ respectively. On the other hand the interaction of $3 \mathbf{a}, \mathbf{b}$ with acetylene derivatives $12 \mathbf{a}, \mathbf{b}, 14$ afforded the corresponding pyrido[2,3-d]pyrimidine $13 \mathbf{a}-\mathbf{d}$ and $\mathbf{1 6 a}$, b respectively. The synthesized 2-azadiene 18 failed to add either electron-poor or push-pull dienophile. The in vitro antimicrobial activity of some of the newly synthesized compounds was examined. All the tested compounds proved to be active as antibacterial and antifungal agents. Also the in vivo antitumor activity of compounds $8 \mathbf{a}, 11 \mathbf{b}, 13 a, d$ and $16 b$ against lung (H460) and liver (HEPG2) carcinoma cells was examined. Compounds 8a, 16b showed moderate activity against lung carcinoma cell line $(\mathrm{H} 460)$.

\section{Keywords}

6-Amino-2-thiouracil, 2-Azadiene, Pyrido[2,3-d]pyrimidine, Antimicrobial, Antitumor. 


\section{Introduction}

Pyrido[2,3-d]pyrimidines are biologically interesting molecules that have established utility in the pharmaceutical and the agrochemical industries. Compounds with these ring systems have diverse pharmacological activity such as antitumor [1,2], cardiotonic [3,4], hepatoprotective [3], antihypertensive [3], antibronchitic [5], antifungal [6], antibacterial [7] and antifolate [8]. Therefore these fused heterocycles have been extensively investigated and their synthetic preparations are well documented [9-11]. As a result, a number of reports appeared in literature; however they usually require forcing conditions [12], long reaction times $[13,14]$ and complex synthetic pathways [2]. So new routes for the synthesis of these molecules have attracted a considerable attention as a rapid entry for the formation of these heterocycles $[15,16]$. This report explains a simple route for synthesis of pyrido[2,3-d] pyrimidine via [4+2] cycloaddition.

\section{Experimental}

All melting points are uncorrected. The IR spectra expressed in $\mathrm{cm}^{-1}$ and recorded in $\mathrm{KBr}$ pellets on a Pa-9721 IR spectrometer. ${ }^{1} \mathrm{H}-\mathrm{NMR},{ }^{13} \mathrm{C}-\mathrm{NMR}$ spectra were obtained on a Varian EM-390 $300 \mathrm{MHz}$ spectrometer in DMSO- $\mathrm{d}_{6}$ as a solvent and TMS as a internal reference. Chemical shifts $(\delta)$ are expressed in ppm. Mass spectra were recorded on Kartos (75 eV) MS equipment. Elemental analyses were carried out by the micro-analytical unit at the National Research Center, Giza, Egypt. Microbiological analyses were carried out by the Micro-analytical Center, Faculty of Science, Cairo University, Giza, Egypt. the antitumor activity was evaluated by the National Cancer Institute, Cancer Biology Department, Cairo University, Egypt. All starting materials used were commercially available by Aldrich company otherwise stated.

\section{Synthesis of 2-azadiene 3a,b:}

To a solution of 6-amino-2-thiouracil $1(1.43 \mathrm{~g}, 0.01 \mathrm{~mol})$ in DMF $(30 \mathrm{ml})$, an equivalent amount of aromatic aldehyde $(0.01 \mathrm{~mol})$ and few drops of acetic acid 
were added. The reaction mixture was heated under reflux for $4 \mathrm{~h}$, then left to cool. The solid product formed after pouring into ice/water was filtered and crystallized from (ethanol/dioxane mixture).

6-(Benzylidene-amino)-2-thioxo-2,3-dihydro-1H-pyrimidin-4-one, 3a: [17] yellow crystals, yield: $2.03 \mathrm{~g}(88 \%)$; mp: $278{ }^{\circ} \mathrm{C}$; IR $\left(\mathrm{KBr}, \mathrm{cm}^{-1}\right): 3386(\mathrm{NH}), 1654$ (CO); ${ }^{1} \mathrm{H}-\mathrm{NMR}$ (DMSO-d $\left.\mathrm{d}_{6}\right): \delta(\mathrm{ppm})=5.26(\mathrm{~s}, 1 \mathrm{H}, \mathrm{H}-5$ pyrimidine), 6.96-7.11 $(\mathrm{m}, 5 \mathrm{H}$, aromatic protons), $7.84(\mathrm{~s}, 1 \mathrm{H}, \mathrm{N}=\mathrm{CH}), 11.37(\mathrm{~s}, 1 \mathrm{H}, \mathrm{NH}), 11.84(\mathrm{~s}, 1 \mathrm{H}, \mathrm{NH}) ; \mathrm{MS}$ $(\mathrm{m} / \mathrm{z})=231\left(\mathrm{M}^{+}, 22 \%\right)$. Anal. Calcd. For $\mathrm{C}_{11} \mathrm{H}_{9} \mathrm{~N}_{3} \mathrm{OS}$ : C $57.13 \%, \mathrm{H} 3.92 \%, \mathrm{~N}$ $18.17 \%$, S $13.86 \%$. Found: C $57.08 \%$, H 3.86\%, N 18.14\%, S $13.78 \%$.

\section{6-[(4-Methoxy-benzylidene)-amino]-2-thioxo-2,3-dihydro-1H-pyrimidin-4-one,} 3b: yellow crystals, yield: $2.38 \mathrm{~g}(91 \%)$; $\mathrm{mp}: 288{ }^{\circ} \mathrm{C}$; IR $\left(\mathrm{KBr}, \mathrm{cm}^{-1}\right): 3387(\mathrm{NH})$, $1656(\mathrm{CO}) ;{ }^{1} \mathrm{H}-\mathrm{NMR}\left(\mathrm{DMSO}_{\mathrm{d}}\right): \delta(\mathrm{ppm})=3.69\left(\mathrm{~s}, 3 \mathrm{H}, \mathrm{OCH}_{3}\right), 5.34(\mathrm{~s}, 1 \mathrm{H}, \mathrm{H}-5$ pyrimidine), $6.77(\mathrm{~d}, 2 \mathrm{H}$, aromatic protons, $\mathrm{J}=8.6 \mathrm{~Hz}), 6.96(\mathrm{~d}, 2 \mathrm{H}$, aromatic protons, J=8.6 Hz), $7.93(\mathrm{~s}, 1 \mathrm{H}, \mathrm{N}=\mathrm{CH}), 11.39(\mathrm{~s}, 1 \mathrm{H}, \mathrm{NH}), 11.93(\mathrm{~s}, 1 \mathrm{H}, \mathrm{NH})$; MS $(\mathrm{m} / \mathrm{z})=262\left(\mathrm{M}^{+}, 20 \%\right)$. Anal. Calcd. For $\mathrm{C}_{12} \mathrm{H}_{11} \mathrm{~N}_{3} \mathrm{O}_{2} \mathrm{~S}: \mathrm{C} 55.16 \%, \mathrm{H} 4.24 \%, \mathrm{~N}$ $16.08 \%$, S $12.27 \%$. Found: C 55.04\%, H 4.18\%, N 15.92\%, S $12.13 \%$.

\section{Cycloaddition reaction of 2-azadiene $3 a, b$ with $\beta$-enaminones and} enaminonitrile:

Equimolar amounts of each of $\mathbf{3 a}, \mathbf{b}(0.01 \mathrm{~mol})$ and one of the $\beta$ - enaminone 4a-c $(0.01 \mathrm{~mol})$ or the enaminonitrile $9(25 \mathrm{ml})$ were heated under reflux in dry dioxane for $16 \mathrm{~h}$. The solvent evaporated under vacuum and the remaining residue was treated with petroleum ether $40-60{ }^{\circ} \mathrm{C}$. The precipitate formed was collected by filtration and crystallized from dioxane.

\section{7-Phenyl-5-(thiophene-2-carbonyl)-2-thioxo-2,3-dihydro-1H-}

pyrido[2,3-]pyrimidin-4-one, 8a: yellow crystals, yield: $2.90 \mathrm{~g}$ (79.4\%); mp: 199 ${ }^{0} \mathrm{C}$; IR (KBr, cm${ }^{-1}$ ): 3327 (NH), 1649 (CO), 1630 (CO); ${ }^{1} \mathrm{H}-\mathrm{NMR}$ (DMSO-d $\mathrm{d}_{6}$ ): $\delta$ 
$(\mathrm{ppm})=7.05-7.24(\mathrm{~m}, 5 \mathrm{H}$, aromatic protons $), 7.63(\mathrm{~s}, 1 \mathrm{H}, \mathrm{H}-6), 7.67-7.75(\mathrm{~m}, 2 \mathrm{H}$, thiophene protons), $8.15(\mathrm{~d}, 1 \mathrm{H}$, thiophene protons, $\mathrm{J}=3 \mathrm{~Hz}), 11.84(\mathrm{~s}, 1 \mathrm{H}, \mathrm{NH})$, $12.07(\mathrm{~s}, 1 \mathrm{H}, \mathrm{NH}) ;{ }^{13} \mathrm{C}-\mathrm{NMR}\left(\mathrm{DMSO}_{\mathrm{d}}\right)$ : $\delta(\mathrm{ppm})=90.86,91.32,125.81,127.01$, 128.33, 128.41, 128.71, 128.93, 131.25 138.0, 146.0, 148.55, 153.98, 163.53 $(\mathrm{C}=\mathrm{O}), 173.42(\mathrm{C}=\mathrm{O}), 179.59(\mathrm{C}=\mathrm{S})$; $\mathrm{MS}(\mathrm{m} / \mathrm{z})=365\left(\mathrm{M}^{+}, 38 \%\right)$. Anal. Calcd. For $\mathrm{C}_{18} \mathrm{H}_{11} \mathrm{~N}_{3} \mathrm{O}_{2} \mathrm{~S}_{2}$ : C $59.16 \%, \mathrm{H} 3.03 \%, \mathrm{~N} 11.50 \%$, S $17.55 \%$. Found: C $59.04 \%, \mathrm{H}$ $2.95 \%$, N $11.47 \%$, S $17.43 \%$.

\section{7-(4-Methoxy-phenyl)-5-(thiophene-2-carbonyl)-2-thioxo-2,3-dihydro-1H-}

pyrido[2,3-d]pyrimidin-4-one, 8b: yellow crystals, yield $3.30 \mathrm{~g}(84 \%)$; mp: $226{ }^{0} \mathrm{C}$; IR $\left(\mathrm{KBr}, \mathrm{cm}^{-1}\right): 3406(\mathrm{NH}), 1646(\mathrm{CO}), 1631(\mathrm{CO}) ;{ }^{1} \mathrm{H}-\mathrm{NMR}\left(\mathrm{DMSO}-\mathrm{d}_{6}\right): \delta(\mathrm{ppm})=$ $3.84\left(\mathrm{~s}, 3 \mathrm{H}, \mathrm{OCH}_{3}\right), 6.75-7.34(\mathrm{~m}, 4 \mathrm{H}$, aromatic protons), $7.63(\mathrm{~s}, 1 \mathrm{H}, \mathrm{H}-6), 7.68-$ $7.98(\mathrm{~m}, 2 \mathrm{H}$, thiophene protons), $8.15(\mathrm{~d}, 1 \mathrm{H}$, thiophene protons, $\mathrm{J}=3 \mathrm{~Hz}), 11.84$ $(\mathrm{s}, 1 \mathrm{H}, \mathrm{NH}), 12.07(\mathrm{~s}, 1 \mathrm{H}, \mathrm{NH})$; MS $(\mathrm{m} / \mathrm{z})=395\left(\mathrm{M}^{+}, 72 \%\right)$. Anal. Calcd. For $\mathrm{C}_{19} \mathrm{H}_{13} \mathrm{~N}_{3} \mathrm{O}_{3} \mathrm{~S}_{2}$ : C $57.71 \%$, H 3.31\%, N 10.63\%, S $16.22 \%$. Found: C $57.67 \%, \mathrm{H}$ $3.26 \%$, N $10.52 \%$, S $16.14 \%$.

\section{5-(Furan-2-carbonyl)-7-phenyl-2-thioxo-2,3-dihydro-1H-pyrido[2,3-d]pyrimidin-}

4-one, 8c: brown crystals, yield: $2.86 \mathrm{~g}(82 \%)$; mp: $212{ }^{\circ} \mathrm{C}$; IR $\left(\mathrm{KBr}, \mathrm{cm}^{-1}\right): 3426$ $(\mathrm{NH}), 1644$ (CO), 1633 (CO); ${ }^{1} \mathrm{H}-\mathrm{NMR}\left(\mathrm{DMSO}_{\mathrm{d}}\right): \delta(\mathrm{ppm})=6.58-7.05(\mathrm{~m}, 5 \mathrm{H}$, aromatic protons), $7.18(\mathrm{~d}, 1 \mathrm{H}$, furan proton, $\mathrm{J}=9 \mathrm{~Hz}), 7.35(\mathrm{~s}, 1 \mathrm{H}, \mathrm{H}-6), 7.48-7.88$ $\left(\mathrm{m}, 2 \mathrm{H}\right.$, furan protons), $11.83(\mathrm{~s}, 1 \mathrm{H}, \mathrm{NH}), 12.06(\mathrm{~s}, 1 \mathrm{H}, \mathrm{NH}) ; \mathrm{MS}(\mathrm{m} / \mathrm{z})=349\left(\mathrm{M}^{+}\right.$, $27 \%$ ). Anal. Calcd. For $\mathrm{C}_{18} \mathrm{H}_{11} \mathrm{~N}_{3} \mathrm{O}_{3} \mathrm{~S}$ : C $61.88 \%, \mathrm{H} 3.17 \%, \mathrm{~N} 12.03 \%$, S 9.18\%. Found: C $61.77 \%$, H 3.08\%, N 11.96\%, S 9.03\%.

\section{5-(Furan-2-carbonyl)-7-(4-methoxy-phenyl)-2-thioxo-2,3-dihydro-1H-}

pyrido[2,3-d]pyrimidin-4-one, 8d: brown crystals, yield: $3.25 \mathrm{~g}$ (85\%); mp: $228{ }^{\circ} \mathrm{C}$; IR $\left(\mathrm{KBr}, \mathrm{cm}^{-1}\right): 3330(\mathrm{NH}), 1640(\mathrm{CO}), 1631(\mathrm{CO}) ;{ }^{1} \mathrm{H}-\mathrm{NMR}\left(\mathrm{DMSO}-\mathrm{d}_{6}\right): \delta(\mathrm{ppm})=$ $3.85\left(\mathrm{~s}, 3 \mathrm{H}, \mathrm{OCH}_{3}\right), 6.76(\mathrm{~d}, 2 \mathrm{H}$, aromatic protons, J= $7.5 \mathrm{~Hz}), 6.96(\mathrm{~d}, 2 \mathrm{H}$, aromatic protons, $\mathrm{J}=7.5), 7.03-7.13(\mathrm{~m}, 1 \mathrm{H}$, furan proton), $7.35(\mathrm{~s}, 1 \mathrm{H}, \mathrm{H}-6), 7.67$ 
(d, $1 \mathrm{H}$, furan proton, $\mathrm{J}=9 \mathrm{~Hz}), 7.86(\mathrm{~d}, 1 \mathrm{H}$, furan proton, $\mathrm{J}=9 \mathrm{~Hz}), 11.83(\mathrm{~s}, 1 \mathrm{H}$, $\mathrm{NH}), 12.05(\mathrm{~s}, 1 \mathrm{H}, \mathrm{NH}) ; \mathrm{MS}(\mathrm{m} / \mathrm{z})=379\left(\mathrm{M}^{+}, 25 \%\right)$. Anal. Calcd. For $\mathrm{C}_{19} \mathrm{H}_{13} \mathrm{~N}_{3} \mathrm{O}_{4} \mathrm{~S}: \mathrm{C}$ $60.15 \%$, H 3.45\%, N 11.08\%, S 8.45\%. Found: C 60.04\%, H 3.32\%, N 10.93\%, S $8.37 \%$.

\section{5-(Naphthalene-2-carbonyl)-7-phenyl-2-thioxo-2,3-dihydro-1H-pyrido[2,3-}

d]pyrimidin-4-one, 8e: yellow crystals, yield: $3.50 \mathrm{~g}(86 \%)$; mp: $233{ }^{\circ} \mathrm{C}$; IR $(\mathrm{KBr}$, $\mathrm{cm}^{-1}$ ): $3399(\mathrm{NH}), 1643$ (CO), 1630 (CO); ${ }^{1} \mathrm{H}-\mathrm{NMR}$ (DMSO-d $\mathrm{d}_{6}$ ): $\delta(\mathrm{ppm})=6.70-7.25$ ( $\mathrm{m}, 5 \mathrm{H}$, aromatic protons), $7.30(\mathrm{~s}, 1 \mathrm{H}, \mathrm{H}-6), 7.54-8.02(\mathrm{~m}, 6 \mathrm{H}$, naphthyl protons), $8.50(\mathrm{~s}, 1 \mathrm{H}$, naphthyl proton), $11.85(\mathrm{~s}, 1 \mathrm{H}, \mathrm{NH}), 12.10(\mathrm{~s}, 1 \mathrm{H}, \mathrm{NH}) ; \mathrm{MS}(\mathrm{m} / \mathrm{z})=409$ $\left(\mathrm{M}^{+}, 43 \%\right)$. Anal. Calcd. For $\mathrm{C}_{24} \mathrm{H}_{15} \mathrm{~N}_{3} \mathrm{O}_{2} \mathrm{~S}$ : C $70.40 \%$, H 3.69\%, N $10.26 \%$, S $7.83 \%$. Found: C $70.33 \%$, H $3.57 \%$, N $10.13 \%$, S $7.79 \%$.

\section{7-(4-Methoxy-phenyl)-5-(naphthalene-2-carbonyl)-2-thioxo-2,3-dihydro-1H-} pyrido[2,3-d]pyrimidin-4-one, 8f: yellow crystals, yield: $3.80 \mathrm{~g}(87 \%)$; mp: $248{ }^{\circ} \mathrm{C}$; IR $\left(\mathrm{KBr}, \mathrm{cm}^{-1}\right)$ : $3408(\mathrm{NH}), 1646(\mathrm{CO}), 1628(\mathrm{CO}) ;{ }^{1} \mathrm{H}-\mathrm{NMR}\left(\mathrm{DMSO}-\mathrm{d}_{6}\right): \delta(\mathrm{ppm})=$ $3.55\left(\mathrm{~s}, 3 \mathrm{H}, \mathrm{OCH}_{3}\right), 6.75(\mathrm{~d}, 2 \mathrm{H}$, aromatic protons, $\mathrm{J}=7.5 \mathrm{~Hz}), 6.94(\mathrm{~d}, 2 \mathrm{H}$, aromatic protons, $\mathrm{J}=7.5 \mathrm{~Hz}$ ), $7.55(\mathrm{~s}, 1 \mathrm{H}, \mathrm{H}-6), 7.56-8.05(\mathrm{~m}, 6 \mathrm{H}$, naphthyl protons), 8.50 (s, $1 \mathrm{H}$, naphthyl protons), $11.85(\mathrm{~s}, 1 \mathrm{H}, \mathrm{NH}), 12.04(\mathrm{~s}, 1 \mathrm{H}, \mathrm{NH}) ; \mathrm{MS}(\mathrm{m} / \mathrm{z})=439\left(\mathrm{M}^{+}\right.$, $27 \%$ ). Anal. Calcd. For $\mathrm{C}_{25} \mathrm{H}_{17} \mathrm{~N}_{3} \mathrm{O}_{3} \mathrm{~S}$ : C $68.32 \%$, H 3.90\%, N 9.56\%, S 7.30\%. Found: C $68.21 \%$, H 3.85\%, N 9.47\%, S 7.23\%.

\section{4-Oxo-7-phenyl-2-thioxo-1,2,3,4-tetrahydropyrido[2,3-d]pyrimidine-5-}

carbonitrile, 11a: brown crystals, yield: $1.90 \mathrm{~g}(70 \%)$; mp: $213^{\circ} \mathrm{C}$; IR $\left(\mathrm{KBr}, \mathrm{cm}^{-1}\right)$ : $3398(\mathrm{NH}), 2203(\mathrm{CN}), 1643(\mathrm{CO}) ;{ }^{1} \mathrm{H}-\mathrm{NMR}\left(\mathrm{DMSO}-\mathrm{d}_{6}\right): \delta(\mathrm{ppm})=6.80-7.23(\mathrm{~m}$, $5 \mathrm{H}$, aromatic protons), 7.38 (s, 1H, H-6), 11.83 (s, 1H, NH), $12.06(\mathrm{~s}, 1 \mathrm{H}, \mathrm{NH}) ; \mathrm{MS}$ $(\mathrm{m} / \mathrm{z})=280\left(\mathrm{M}^{+}, 7 \%\right)$. Anal. Calcd. For $\mathrm{C}_{14} \mathrm{H}_{8} \mathrm{~N}_{4} \mathrm{OS}$ : C 59.99\%, H 2.88\%, N 19.99\%, S 11.44\%. Found: C 59.87\%, H 2.75\%, N 19.87\%, S 11.38\%. 


\section{7-(4-Methoxy-phenyl)-4-oxo-2-thioxo-1,2,3,4-tetrahydro-pyrido[2,3-}

d]pyrimidine-5-carbonitrile, 11b: brown crystals, yield: $2.30 \mathrm{~g}(76 \%)$; $\mathrm{mp}: 228{ }^{0} \mathrm{C}$; IR $\left(\mathrm{KBr}, \mathrm{cm}^{-1}\right): 3401(\mathrm{NH}), 2182(\mathrm{CN}), 1649(\mathrm{CO}) ;{ }^{1} \mathrm{H}-\mathrm{NMR}\left(\mathrm{DMSO}-\mathrm{d}_{6}\right): \delta(\mathrm{ppm})=$ $3.55\left(\mathrm{~s}, 3 \mathrm{H}, \mathrm{OCH}_{3}\right), 6.75(\mathrm{~d}, 2 \mathrm{H}$, aromatic protons, $\mathrm{J}=7.5 \mathrm{~Hz}), 6.94(\mathrm{~d}, 2 \mathrm{H}$, aromatic protons, J= 7.5 Hz), $7.35(\mathrm{~s}, 1 \mathrm{H}, \mathrm{H}-6), 11.83(\mathrm{~s}, 1 \mathrm{H}, \mathrm{NH}), 12.06(\mathrm{~s}, 1 \mathrm{H}, \mathrm{NH}) ;{ }^{13} \mathrm{C}-$ NMR $\left(D M S O-d_{6}\right): \delta(p p m)=55.52\left(\mathrm{OCH}_{3}\right), 90.42,91.12,113.70,113.84(\mathrm{CN})$, 128.05, 128.82, 130.21, 142.0, 153.96, 157.69, 163.50 (C=O), $173.37(\mathrm{C}=\mathrm{S})$; MS $(\mathrm{m} / \mathrm{z})=310\left(\mathrm{M}^{+}, 8 \%\right)$. Anal. Calcd. For $\mathrm{C}_{15} \mathrm{H}_{10} \mathrm{~N}_{4} \mathrm{O}_{2} \mathrm{~S}: \mathrm{C} 58.06 \%, \mathrm{H} 3.25 \%, \mathrm{~N}$ $18.05 \%$, S 10.33\%. Found: C 57.92\%, H 3.12\%, N 17.96\%, S 10.24\%.

\section{Cycloaddition reaction of 2-azadiene 3a,b with acetylenes:}

To a solution of each of 2-azadiene $\mathbf{3 a}, \mathbf{b}(0.01 \mathrm{~mol})$ (in dry dioxane $(30 \mathrm{ml})$ ), equimolar amounts of one of the acetylenes $12 \mathbf{a}, \mathbf{b}$ or 14 was added. The reaction mixture was heated under reflux for $4 \mathrm{~h}$. The solvent was evaporated under vacuum. After treaturation of the remaining residue with $n$-hexane; the solid product formed was collected by filtration and crystallized from dioxane.

\section{Diethyl 4-oxo-7-phenyl-2-thioxo-1,2,3,4-tetrahydropyrido[2,3-d]pyrimidine-5,6-} dicarboxylate, 13a: orange crystals, yield: $3.50 \mathrm{~g}(87 \%)$; mp: $235^{\circ} \mathrm{C}$; IR $\left(\mathrm{KBr}, \mathrm{cm}^{-}\right.$ ${ }^{1}$ ): $3419(\mathrm{NH}), 1760$ (CO), 1726 (CO), 1633 (CO); ${ }^{1} \mathrm{H}-\mathrm{NMR}$ (DMSO-d 6 ): $\delta$ (ppm)= 1.13-1.29 (m, $\left.6 \mathrm{H}, 2 \mathrm{CH}_{3}\right), 4.13-4.28\left(\mathrm{~m}, 4 \mathrm{H}, 2 \mathrm{CH}_{2}\right), 6.77-7.21(\mathrm{~m}, 5 \mathrm{H}$, aromatic protons), $11.85(\mathrm{~s}, 1 \mathrm{H}, \mathrm{NH}), 12.05(\mathrm{~s}, 1 \mathrm{H}, \mathrm{NH}) ;{ }^{13} \mathrm{C}-\mathrm{NMR}\left(\mathrm{DMSO}_{6} \mathrm{~d}_{6}\right): \delta(\mathrm{ppm})=$ 14.02, $14.50\left(2 \mathrm{CH}_{3}\right), 62.49,66.87\left(2 \mathrm{CH}_{2}\right), 109.40,119.10,126.89,127.07,127.90$, 128.29, 142.0, 156.0, 161.53, 163.0 ( $\mathrm{C}=\mathrm{O}), 166.01$ ( $\mathrm{C}=\mathrm{O}), 166.80(\mathrm{C}=\mathrm{O}), 172.90$ $(\mathrm{C}=\mathrm{S}) ; \mathrm{MS}(\mathrm{m} / \mathrm{z})=399\left(\mathrm{M}^{+}, 9 \%\right), 354\left(\mathrm{M}^{+}-\mathrm{OEt}, 9 \%\right)$. Anal. Calcd. for $\mathrm{C}_{19} \mathrm{H}_{17} \mathrm{~N}_{3} \mathrm{O}_{5} \mathrm{~S}$ : C $57.13 \%$, H $4.29 \%$, N $10.52 \%$, S $8.03 \%$. Found: C $57.04 \%$, H $4.23 \%$, N $10.43 \%$, S $7.9 \%$.

\section{Diethyl 7-(4-methoxyphenyl)-4-oxo-2-thioxo-1,2,3,4-tetrahydro-pyrido[2,3-d]-} pyrimidine-5,6-dicarboxylate, 13b: orange crystals, yield: $3.80 \mathrm{~g}(88 \%)$; $\mathrm{mp}: 246$ ${ }^{0} \mathrm{C}$; IR (KBr, cm ${ }^{-1}$ ): 3426 (NH), 1762 (CO), 1733 (CO), 1637 (CO); ${ }^{1} \mathrm{H}-\mathrm{NMR}$ (DMSO- 
$\left.\mathrm{d}_{6}\right): \delta(\mathrm{ppm})=1.13-1.29\left(\mathrm{~m}, 6 \mathrm{H}, 2 \mathrm{CH}_{3}\right), 3.69\left(\mathrm{~s}, 3 \mathrm{H}, \mathrm{OCH}_{3}\right), 4.13-4.45(\mathrm{~m}, 4 \mathrm{H}$, $\left.2 \mathrm{CH}_{2}\right), 6.77-6.98(\mathrm{~m}, 4 \mathrm{H}$, aromatic protons), $11.82(\mathrm{~s}, 1 \mathrm{H}, \mathrm{NH}), 12.01(\mathrm{~s}, 1 \mathrm{H}, \mathrm{NH})$; MS $(\mathrm{m} / \mathrm{z})=429\left(\mathrm{M}^{+}, 3 \%\right), 384\left(\mathrm{M}^{+}-\mathrm{OEt}, 61 \%\right)$. Anal. Calcd. for $\mathrm{C}_{20} \mathrm{H}_{19} \mathrm{~N}_{3} \mathrm{O}_{6} \mathrm{~S}: \mathrm{C}$ $55.94 \%, \mathrm{H} 4.46 \%$, N 9.78\%, S 7.47\%. Found: C 55.86\%, H 4.34\%, N 9.69\%, S $7.36 \%$.

Dimethyl 4-oxo-7-phenyl-2-thioxo-1,2,3,4-tetrahydro-pyrido[2,3-d]pyrimidine5,6-dicarboxylate, 13c: yellow crystals, yield: $3.20 \mathrm{~g}(86 \%)$; mp: $200{ }^{\circ} \mathrm{C}$; IR $(\mathrm{KBr}$, $\mathrm{cm}^{-1}$ ) $3426(\mathrm{NH}), 1762(\mathrm{CO}), 1732(\mathrm{CO}), 1636(\mathrm{CO}) ;{ }^{1} \mathrm{H}-\mathrm{NMR}\left(\mathrm{DMSO}-\mathrm{d}_{6}\right): \delta(\mathrm{ppm})=$ $3.72\left(\mathrm{~s}, 3 \mathrm{H}, \mathrm{CH}_{3}\right), 3.79\left(\mathrm{~s}, 3 \mathrm{H}, \mathrm{CH}_{3}\right), 7.01-7.38(\mathrm{~m}, 5 \mathrm{H}$, aromatic protons), $11.80(\mathrm{~s}$, $1 \mathrm{H}, \mathrm{NH}), 12.05(\mathrm{~s}, 1 \mathrm{H}, \mathrm{NH}) ; \mathrm{MS}(\mathrm{m} / \mathrm{z})=371\left(\mathrm{M}^{+}, 2 \%\right), 356\left(\mathrm{M}^{+}-\mathrm{Me}, 3 \%\right)$. Anal. Calcd. for $\mathrm{C}_{17} \mathrm{H}_{13} \mathrm{~N}_{3} \mathrm{O}_{5} \mathrm{~S}$ : C $54.98 \%, \mathrm{H} 3.53 \%, \mathrm{~N} 11.31 \%$, S 8.63\%. Found: C $54.87 \%, \mathrm{H} 3.44 \%$, N $11.27 \%$, S $8.57 \%$.

\section{Dimethyl 7-(4-methoxyphenyl)-4-oxo-2-thioxo-1,2,3,4-tetrahydro-pyrido[2,3-} d]pyrimidine-5,6-dicarboxylate, 13d: yellow crystals, yield $3.60 \mathrm{~g}$ (89\%); mp: 220 ${ }^{0} \mathrm{C}$; IR (KBr, cm ${ }^{-1}$ ): $3425(\mathrm{NH}), 1763$ (CO), 1733 (CO), 1642 (CO); ${ }^{1} \mathrm{H}-\mathrm{NMR}$ (DMSO$\left.\mathrm{d}_{6}\right): \delta(\mathrm{ppm})=3.58\left(\mathrm{~s}, 3 \mathrm{H}, \mathrm{OCH}_{3}\right), 3.72\left(\mathrm{~s}, 3 \mathrm{H}, \mathrm{CH}_{3}\right), 3.84\left(\mathrm{~s}, 3 \mathrm{H}, \mathrm{CH}_{3}\right), 7.01-7.38$ (m, 4H, aromatic protons), $11.85(\mathrm{~s}, 1 \mathrm{H}, \mathrm{NH}), 12.05(\mathrm{~s}, 1 \mathrm{H}, \mathrm{NH}) ; \mathrm{MS}(\mathrm{m} / \mathrm{z})=401$ $\left(\mathrm{M}^{+}, 2 \%\right), 386\left(\mathrm{M}^{+}-\mathrm{Me}, 20 \%\right)$. Anal. Calcd. for $\mathrm{C}_{18} \mathrm{H}_{15} \mathrm{~N}_{3} \mathrm{O}_{6} \mathrm{~S}: \mathrm{C} 53.86 \%, \mathrm{H} 3.77 \%, \mathrm{~N}$ $10.47 \%$, S 7.99\%. Found: C 53.79\%, H 3.63\%, N 10.34\%, S 7.82\%.

\section{Ethyl 4-oxo-7-phenyl-2-thioxo-1,2,3,4-tetrahydropyrido[2,3-d]pyrimidine-5-} carboxylate, 16a: yellow crystals, yield: $2.65 \mathrm{~g}(81 \%)$; mp: $242{ }^{\circ} \mathrm{C}$; IR $\left(\mathrm{KBr}, \mathrm{cm}^{-1}\right)$ : $3407(\mathrm{NH}), 1761$ (CO), 1644 (CO); ${ }^{1} \mathrm{H}-\mathrm{NMR}$ (DMSO-d $\mathrm{d}_{6}$ ): $\delta(\mathrm{ppm})=1.21\left(\mathrm{t}, 3 \mathrm{H}, \mathrm{CH}_{3}\right.$, $\mathrm{J}=7 \mathrm{~Hz}$ ), 4.14 (q, 2H, $\mathrm{CH}_{2}, \mathrm{~J}=7 \mathrm{~Hz}$ ), 7.05-7.20 (m, 5H, aromatic protons), 7.38 (s, $1 \mathrm{H}, \mathrm{H}-6), 11.85(\mathrm{~s}, 1 \mathrm{H}, \mathrm{NH}), 12.05(\mathrm{~s}, 1 \mathrm{H}, \mathrm{NH}) ; \mathrm{MS}(\mathrm{m} / \mathrm{z})=327\left(\mathrm{M}^{+}, 2 \%\right), 254\left(\mathrm{M}^{+}\right.$COOEt, 6\%). Anal. Calcd. for $\mathrm{C}_{16} \mathrm{H}_{13} \mathrm{~N}_{3} \mathrm{O}_{3} \mathrm{~S}$ : C $58.70 \%, \mathrm{H} 4.00 \%, \mathrm{~N} 12.84 \%$, S 9.79\%. Found: C 58.61\%, H 3.96\%, N 12.76\%, S 9.68\%. 


\section{Ethyl 7-(4-methoxyphenyl)-4-oxo-2-thioxo-1,2,3,4-tetrahydro-pyrido[2,3-} d]pyrimidine-5-carboxylate, 16b: yellow crystals, yield: $3.0 \mathrm{~g}(84 \%) ; \mathrm{mp}: 258{ }^{\circ} \mathrm{C}$; IR (KBr, cm $\left.{ }^{-1}\right) 3414(\mathrm{NH}), 1764$ (CO), 1646 (CO); ${ }^{1} \mathrm{H}-\mathrm{NMR}\left(\mathrm{DMSO}-\mathrm{d}_{6}\right): \delta(\mathrm{ppm})=$ $1.23\left(\mathrm{t}, 3 \mathrm{H}, \mathrm{CH}_{3}, \mathrm{~J}=7.2 \mathrm{~Hz}\right), 3.69\left(\mathrm{~s}, 3 \mathrm{H}, \mathrm{OCH}_{3}\right), 4.15\left(\mathrm{q}, 2 \mathrm{H}, \mathrm{CH}_{2}, \mathrm{~J}=7.2 \mathrm{~Hz}\right), 6.76$ (d, $2 \mathrm{H}$, aromatic protons, $\mathrm{J}=7.5 \mathrm{~Hz}), 6.93(\mathrm{~d}, 2 \mathrm{H}$, aromatic protons, $\mathrm{J}=7.5 \mathrm{~Hz}), 7.35$ (s, 1H, H-6), $11.80(\mathrm{~s}, 1 \mathrm{H}, \mathrm{NH}), 11.99(\mathrm{~s}, 1 \mathrm{H}, \mathrm{NH}) ;{ }^{13} \mathrm{C}-\mathrm{NMR}\left(\mathrm{DMSO}_{-} \mathrm{d}_{6}\right): \delta(\mathrm{ppm})=$ $14.08\left(\mathrm{CH}_{3}\right), 54.89\left(\mathrm{OCH}_{3}\right), 66.31\left(\mathrm{CH}_{2}\right), 90.48,94.01,113.23,127.33,127.46$, 129.49, 153.30, 157.06, 160.60, $162.90(\mathrm{C}=\mathrm{O}), 164.04(\mathrm{C}=\mathrm{O}), 172.70(\mathrm{C}=\mathrm{S})$; MS $(\mathrm{m} / \mathrm{z})=357\left(\mathrm{M}^{+}, 1 \%\right), 328\left(\mathrm{M}^{+}-\mathrm{C}_{2} \mathrm{H}_{5}, 26 \%\right)$. Anal. Calcd. for $\mathrm{C}_{17} \mathrm{H}_{15} \mathrm{~N}_{3} \mathrm{O}_{4} \mathrm{~S}: \mathrm{C}$ $57.13 \%$, H $4.23 \%$, N $11.76 \%$, S 8.97\%. Found: C $57.04 \%$, H 4.17\%, N $11.60 \%$, S $8.89 \%$.

\section{Synthesis of dithioimidocarbmate 18:}

A mixture of equimolar amounts of 6-amino-2-thiouracil $1(1.43 \mathrm{~g}, 0.01 \mathrm{~mol})$, $\mathrm{CS}_{2}(0.01 \mathrm{~mol})$ and sodium hydride $(0.48 \mathrm{~g}, 0.02 \mathrm{~mol})$ in DMSO $(50 \mathrm{ml})$ were stirred in ice bath for $3 \mathrm{~h}$. The non-isolable disodium salt 17 was treated with Mel $(2.84 \mathrm{~g}$, $0.02 \mathrm{~mol}$ ). The reaction mixture was stirred for another $3 \mathrm{~h}$ at room temperature then poured over ice/water. The solid product formed was collected by filtration and crystallized from (ethanol/dioxane mixture).

\section{Dimethyl 6-oxo-2-thioxo-1,2,3,6-tetrahydropyrimidin-4-yldithioimido-} carbonate, 18: yellow crystals, yield: $2.10 \mathrm{~g}(85 \%)$; $\mathrm{mp}: 230{ }^{\circ} \mathrm{C}$; $\mathrm{IR}\left(\mathrm{KBr}, \mathrm{cm}^{-1}\right)$ : $3463(\mathrm{NH}), 1640$ (CO); ${ }^{1} \mathrm{H}-\mathrm{NMR}$ (DMSO-d $\left.\mathrm{d}_{6}\right): \delta(\mathrm{ppm})=2.49\left(\mathrm{~s}, 3 \mathrm{H}, \mathrm{CH}_{3}\right), 2.76(\mathrm{~s}$, $\left.3 \mathrm{H}, \mathrm{CH}_{3}\right), 6.39\left(\mathrm{~s}, 1 \mathrm{H}, \mathrm{H}-5\right.$ pyrimidine), 11.80 (brs, 2H, 2NH); $\mathrm{MS}(\mathrm{m} / \mathrm{z})=247\left(\mathrm{M}^{+}\right.$, $45 \%$ ). Anal. Calcd. for $\mathrm{C}_{7} \mathrm{H}_{9} \mathrm{~N}_{3} \mathrm{OS}_{3}$ : C 33.99\%, H 3.67\%, N 16.99\%, S 38.89\%. Found: C $33.83 \%$, H $3.55 \%$, N $16.82 \%$, S 38.76\%.

\section{Bioassay}

1. Antimicrobial activity: A filter paper sterilized disc saturated with measured quantity of the sample is placed on plate containing solid bacterial medium (nutrient 
agar broth) or fungal medium (Dox's medium) which has been heavily seeded with spore suspension of the tested organism. After inoculation, the diameter of the clear zone of inhibition surrounding the sample is taken as a measure of the inhibitory power of the sample against the particular test organism.[18-20]

\section{Antitumor activity}

Potential cytotoxicity of the compounds was tested using the method of Skehan et al.[21] Cells were plated in 96 multiwell plate (104 cells/well) for 24 hours before treatment with the compounds to allow attachment of cell to the wall of the plate. Different concentrations of the compound under test $(0.0,1.0,2.5,5.0$ and $10.0 \mu \mathrm{g} / \mathrm{ml}$ ) were added to the cell monolayer. Triplicate wells were prepared for each individual dose. Monolayer cells were incubated with the compounds for 48 hours at $37^{\circ} \mathrm{C}$ and in atmosphere of $5 \% \mathrm{CO}_{2}$. After 48 hours, cells were fixed, washed and stained with sulforhodamine B stain. Excess stain was washed with acetic acid and attached stain was recovered with Tris EDTA buffer. Color intensity was measured in an ELISA reader. The relation between surviving fraction and drug concentrations is plotted to get the survival curve of each tumor cell line after specified compound. The efficiency of the cytotoxic activity is expressed as $I_{50}$.

\section{Results and Discussion}

\section{Chemistry}

6-(Benzylidene-amino)-2-thiouracil $\mathbf{3 a}$ was synthesized previously [17]. However, in this report a simple condensation reaction of 6 -amino-2-thioxo-1Hpyrimidine-4-one 1 [22] with aromatic aldehydes in DMF with few drops of acetic acid afforded the corresponding condensation products 2 or 3 . The formation of the isomeric imino compound $\mathbf{2}$ is not plausible because the imino group is much more nucleophilic than the $\mathrm{CH}$ in position 5 of thiouracil 1 . In addition the ${ }^{1} \mathrm{H}-\mathrm{NMR}$ showed one proton signal at $\delta=5.26 \mathrm{ppm}$ for pyrimidine $\mathrm{H}-5$ and one proton signal at $\delta=7.84 \mathrm{ppm}$ for azamethine proton $(\mathrm{N}=\mathrm{CH})$. That established structure 3 (Scheme 1). 

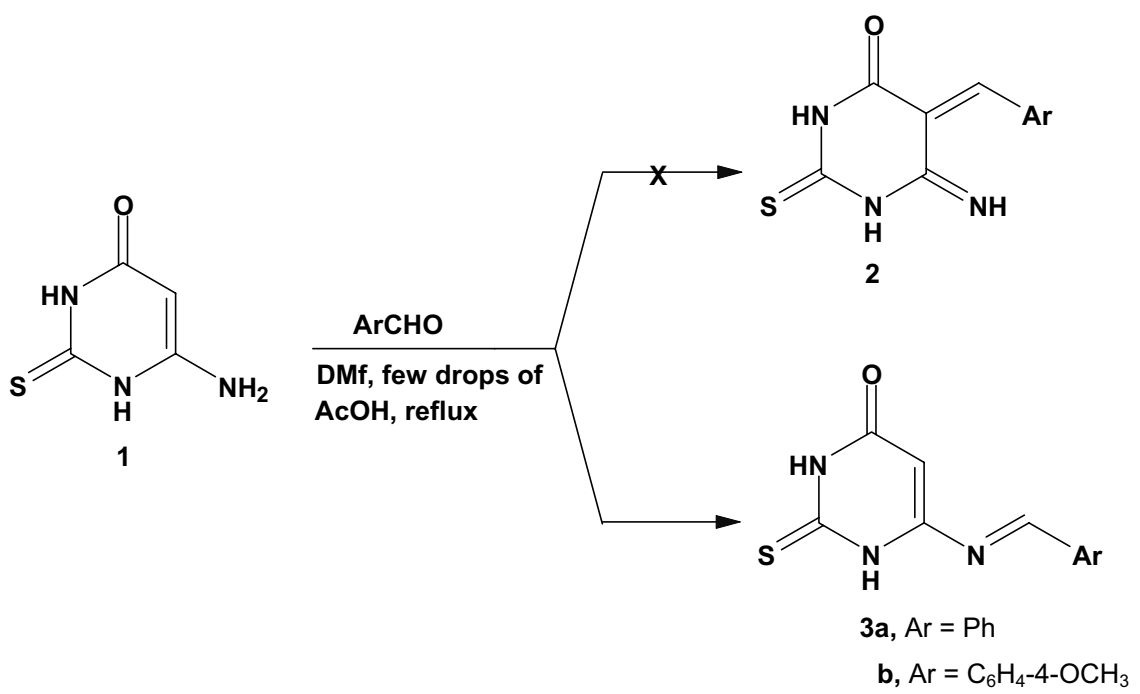

Scheme 1

We looked at the activity of this synthesized diene system in cycloaddition reaction. It has been found that enaminones $\mathbf{4 a - c}$ [23] readily condensed with $\mathbf{3 a , b}$ to yield pyrido[2,3-d]pyrimidine via dimethylamine elimination. One can assume that a [4+2] cycloaddition initially occurred that was followed by secondary amine elimination. This would lead to 7 or isomeric 8 . Structure 8 could be established based on ${ }^{1} \mathrm{H}$-NMR. For example, the ${ }^{1} \mathrm{H}-\mathrm{NMR}$ of compound $8 \mathrm{a}$ revealed a singlet at $\delta=7.63 \mathrm{ppm}$ typical for $\mathrm{H}-6$ proton. This value is different than the expected value for $\mathrm{H}-5$ proton that expected to appear at $\delta=8.40 \mathrm{ppm}$ [24] (Scheme 2).

Similarly, we examined the cycloaddition reaction of the azadiene $\mathbf{3 a}, \mathbf{b}$ with enaminonitrile 9 [25]. The condensed products were formed via piperidine elimination to yield the corresponding 10 or 11. As an example the structure of 11a was established depending on ${ }^{1} \mathrm{H}-\mathrm{NMR}$ spectrum that revealed a singlet at $\delta=7.38$ ppm for pyridopyrimidine $\mathrm{H}-6$. If the reaction product was the isomeric 10; then proton H-5 should appear at a much lower field [24] (Scheme 3). 

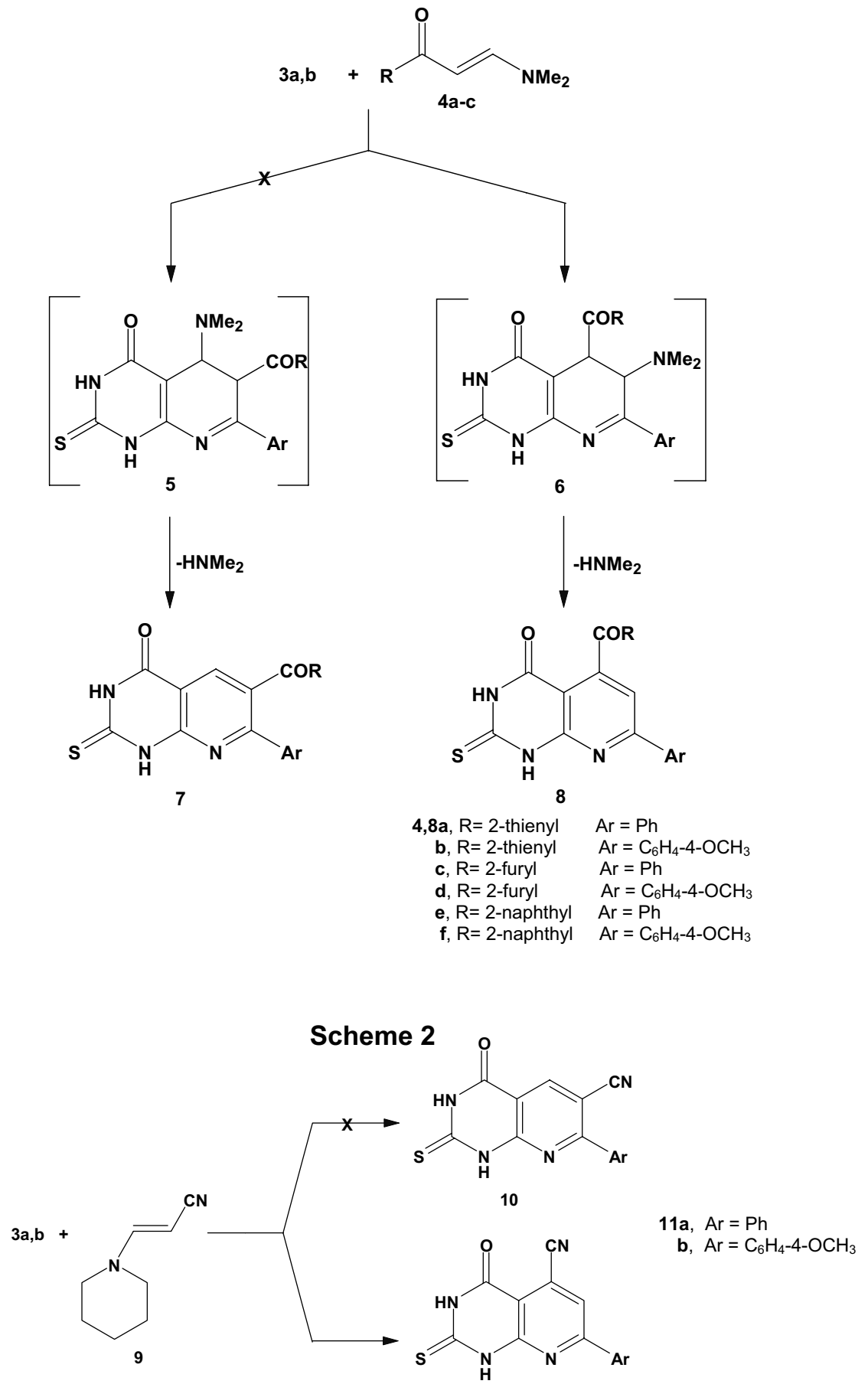

Scheme 3 
In addition compounds $\mathbf{3} \mathbf{a}, \mathbf{b}$ could be successfully added to dimethyl acetylenedicarboxylate and diethyl acetylenedicarboxylate $12 \mathbf{a}, \mathbf{b}$ in dioxane under reflux for 4 hours to produce 13a-d in good yield (Scheme 4).

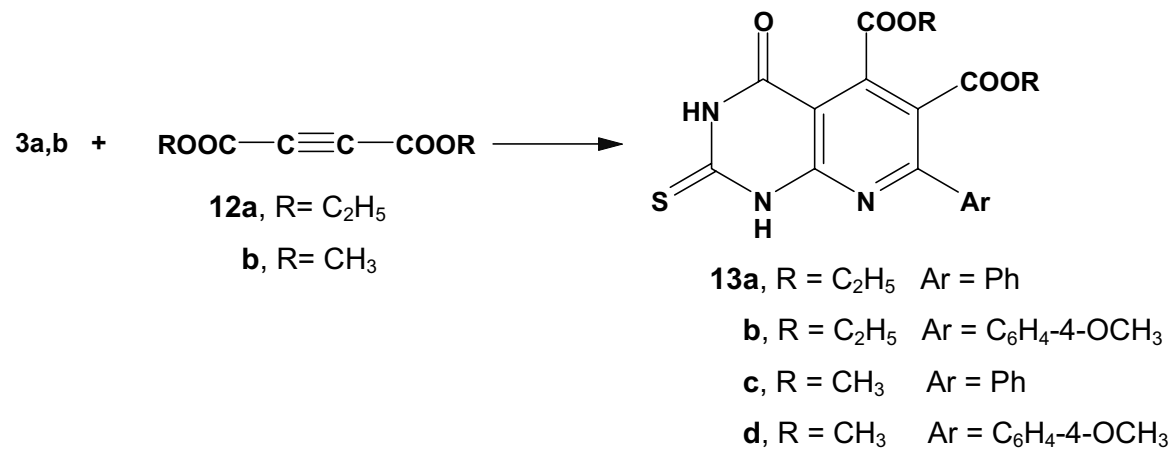

\section{Scheme 4}

Similarly, the reaction of $\mathbf{3 a , b}$ with ethyl propynoate $\mathbf{1 4}$ produced pyrido [2,3-d]pyrimidine 15 or its isomeric 16, that revealed the aryl and ester functions not to be especially proximal (Scheme 5). Structure 16 could be established based on ${ }^{1} \mathrm{H}$-NMR. For example, the ${ }^{1} \mathrm{H}-\mathrm{NMR}$ spectrum of $16 \mathrm{a}$ showed the presence of $\mathrm{H}-6$ proton as singlet at $\delta=7.38 \mathrm{ppm}$. In contrast $\mathrm{H}-5$ proton should appear at a lower field that expected at (ca. $\delta=8.40 \mathrm{ppm}$ ) [24] (cf. experimental section).

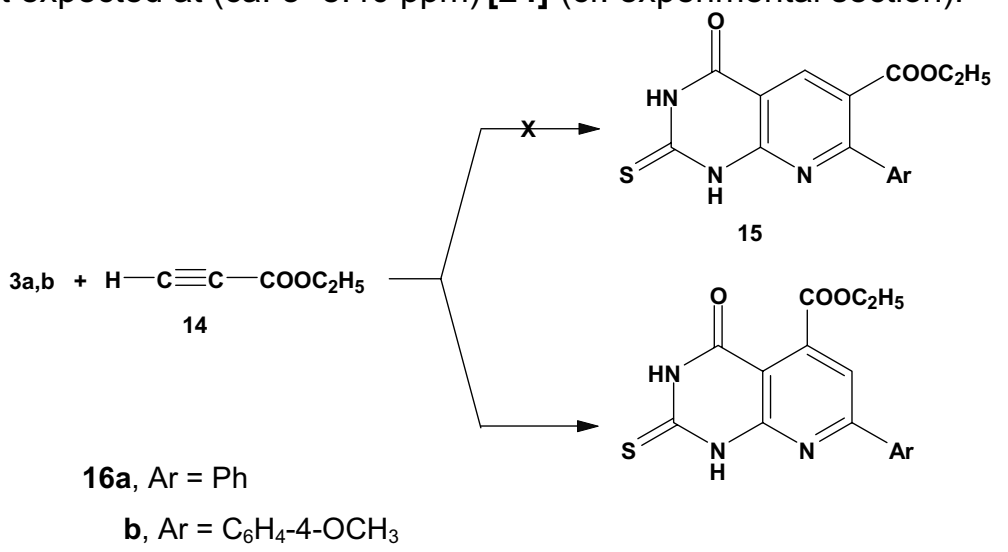

Scheme 5 
Finally, other azadiene system could also be prepared via reacting 1 with $\mathrm{CS}_{2}$ in DMSO in the presence of $\mathrm{NaH}$ forming the non-isolable disodium salt 17 , which reacted with methyl iodide to produce the diene system 18 . This system failed to add either electron-poor or push-pull dienophile (Scheme 6).

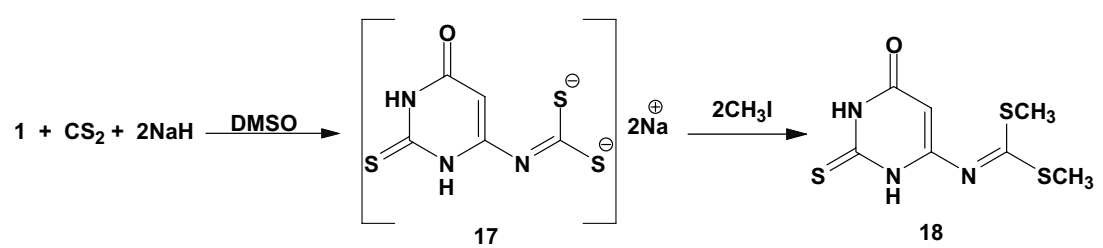

\section{Scheme 6}

In conclusion a simple route to azadiene could be developed and the reactivity of these azadiene systems in Diels-Alder cycloaddition has been investigated.

\section{Bioactivity}

\subsection{Antimicrobial activity}

The in vitro antimicrobial activity of the newly synthesized compounds 8a,b,d,e, 11a, 13a,c,d and 16a against of three strains of Gram positive bacteria, three strains of Gram negative bacteria and two strains of fungi was investigated in comparison with Ampicillin and Nystatine. In general all tested compounds were capable of inhibiting the growth of the all tested strains. Compounds $\mathbf{8 a}$ and $\mathbf{1 3 c , d}$ showed a relatively high activity as antibacterial and antifungal activity agents. The other compounds showed a relatively moderate activity toward the all tested strains. Table (1) shows the results of the bioassay. 


\begin{tabular}{|c|c|c|c|c|c|c|c|c|c|c|c|}
\hline \multirow{2}{*}{ Microorganism } & \multicolumn{11}{|c|}{ Compounds } \\
\hline & $8 a$ & $8 b$ & $8 d$ & $8 \mathrm{e}$ & $11 a$ & $13 \mathbf{a}$ & $13 c$ & 13d & $16 a$ & Amp. & Nys. \\
\hline $\begin{array}{c}\text { Bacillus Subtilis } \\
\left(G^{+}\right)\end{array}$ & 13 & 11 & 11 & 10 & 11 & 12 & 16 & 14 & 11 & 18 & - \\
\hline $\begin{array}{l}\text { Staphylococcus } \\
\text { Aureus }\left(G^{+}\right)\end{array}$ & 12 & 11 & 11 & 11 & 11 & 12 & 16 & 15 & 11 & 20 & - \\
\hline $\begin{array}{l}\text { Streptococcus } \\
\text { Faecalis }\left(G^{+}\right)\end{array}$ & 12 & 12 & 11 & 11 & 12 & 12 & 15 & 14 & 11 & 30 & - \\
\hline $\begin{array}{c}\text { Escherichia Coli } \\
\left(G^{\prime}\right)\end{array}$ & 13 & 12 & 11 & 10 & 12 & 11 & 16 & 14 & 11 & 11 & - \\
\hline $\begin{array}{c}\text { Neisseria } \\
\text { gonorrhea (G') }\end{array}$ & 13 & 14 & 12 & 12 & 12 & 13 & 15 & 14 & 12 & 13 & - \\
\hline $\begin{array}{c}\text { Pseudomonas } \\
\text { Aeroginosa } \\
\text { (G') }\end{array}$ & 12 & 11 & 11 & 11 & 11 & 13 & 16 & 14 & 11 & 19 & - \\
\hline $\begin{array}{l}\text { Candida } \\
\text { Albicans } \\
\text { (Fungus) }\end{array}$ & 13 & 12 & 11 & 10 & 12 & 12 & 15 & 14 & 11 & - & 12 \\
\hline $\begin{array}{c}\text { Saccharomyces } \\
\text { Cereviseae } \\
\text { (Fungus) }\end{array}$ & 12 & 12 & 12 & 12 & 12 & 11 & 16 & 14 & 12 & - & 11 \\
\hline
\end{tabular}

$\mathrm{G}^{+}=$Gram positive Amp. $=$ Ampicillin $\mathrm{G}^{-}=$Gram negative Nys. $=$ Nystatine

Table 1. Antimicrobial potentialities of the tested compounds expressed as size $(\mathrm{mm} / \mathrm{mg}$ sample) of inhibition zone.

\subsection{Antitumor activity}

Evaluation of the anticancer activity of compounds 8a, 11b, 13a,d and 16b was performed at the National Cancer Institute $(\mathrm{NCl})$. The tested compounds were evaluated for cytotoxicity against the liver carcinoma cell line (HEPG2) and the lung carcinoma cell line $(\mathrm{H} 460)$ of human. Different concentrations of the tested compounds were added to the cell monolayer of tumor. A 48 hours continuous drug 
exposure is used to estimate all availability or growth.[21] The cytotoxic activity of each compound is deduced from the dose response curves. Table (2) and (3) represent the cytotoxic activity for each concentration of the tested compounds.

\begin{tabular}{|c|c|c|c|}
\hline \multirow{3}{*}{ Compound } & \multirow{2}{*}{ Conc. \% $\mathbf{\mu g}$} & \multicolumn{2}{|c|}{ HEPG2 } \\
\cline { 3 - 4 } & 0.0 & Y & SEM \\
\hline \multirow{4}{*}{$\mathbf{8 a}$} & 1.0 & 1.000 & \pm 0.033 \\
& 2.5 & 1.050 & \pm 0.008 \\
& 5.0 & 0.905 & \pm 0.012 \\
& 10.0 & 0.850 & \pm 0.012 \\
& 0.0 & 1.000 & \pm 0.020 \\
\hline \multirow{4}{*}{ 11b } & 1.0 & 1.046 & \pm 0.012 \\
& 2.5 & 0.968 & \pm 0.008 \\
& 5.0 & 0.941 & \pm 0.008 \\
& 10.0 & 0.914 & \pm 0.008 \\
\hline \multirow{4}{*}{ 13a } & 0.0 & 1.000 & \pm 0.033 \\
& 1.0 & 0.968 & \pm 0.008 \\
& 2.5 & 0.914 & \pm 0.014 \\
& 5.0 & 0.841 & \pm 0.012 \\
& 10.0 & 0.805 & \pm 0.008 \\
\hline 13d & 0.0 & 1.000 & \pm 0.033 \\
& 1.0 & 0.895 & \pm 0.024 \\
& 2.5 & 0.823 & \pm 0.012 \\
& 5.0 & 0.786 & \pm 0.012 \\
& 10.0 & 0.709 & \pm 0.008 \\
\hline 16b & 0.0 & 1.000 & \pm 0.033 \\
& 1.0 & 1.018 & \pm 0.020 \\
& 2.5 & 1.000 & \pm 0.009 \\
& 5.0 & 0.977 & \pm 0.012 \\
& 10.0 & 0.927 & \pm 0.008 \\
\hline
\end{tabular}

$\mathrm{Y}=$ Surviving fraction, $\mathrm{SEM}=$ Standard deviation .

Table 2. Cytotoxic activity against HEPG2. 


\begin{tabular}{|c|c|c|c|}
\hline \multirow{2}{*}{ Compound } & \multirow{2}{*}{ Conc. \% $\mathbf{\mu g}$} & \multicolumn{2}{|c|}{ H460 } \\
\cline { 2 - 4 } & 0.0 & $\mathbf{Y}$ & SEM \\
\hline \multirow{4}{*}{$\mathbf{8 a}$} & 1.0 & 1.000 & \pm 0.033 \\
& 2.5 & 0.943 & \pm 0.012 \\
& 5.0 & 0.869 & \pm 0.020 \\
& 10.0 & 0.808 & \pm 0.008 \\
$\mathbf{1 1 b}$ & 0.0 & 0.641 & \pm 0.021 \\
\hline & 1.0 & 0.948 & \pm 0.033 \\
& 2.5 & 0.897 & \pm 0.008 \\
& 5.0 & 0.836 & \pm 0.017 \\
& 10.0 & 0.808 & \pm 0.008 \\
\hline 13a & 0.0 & 1.000 & \pm 0.033 \\
& 1.0 & 0.855 & \pm 0.009 \\
& 2.5 & 0.827 & \pm 0.012 \\
& 5.0 & 0.771 & \pm 0.012 \\
13d & 10.0 & 0.720 & \pm 0.005 \\
\hline & 0.0 & 1.000 & \pm 0.033 \\
& 1.0 & 0.962 & \pm 0.008 \\
& 2.5 & 0.887 & \pm 0.012 \\
& 5.0 & 0.808 & \pm 0.008 \\
16b & 10.0 & 0.743 & \pm 0.012 \\
\hline & 0.0 & 1.000 & \pm 0.033 \\
& 1.0 & 0.948 & \pm 0.020 \\
& 2.5 & 0.915 & \pm 0.009 \\
& 5.0 & 0.776 & \pm 0.012 \\
& 10.0 & 0.646 & \pm 0.008 \\
\hline
\end{tabular}

$\mathrm{Y}=$ Surviving fraction, $\mathrm{SEM}=\mathrm{Standard}$ deviation.

Table 3. Cytotoxic activity against $\mathrm{H} 460$.

\section{Conclusion}

All the tested compounds showed limited cytotoxic activity against (HEPG2). Their efficiency is ranged from $10-30 \%$ only. On the other hand, the cytotoxic activity against $(\mathrm{H} 460)$ is more localized and ranged from $20-36 \%$. Compounds $8 \mathbf{a}$ and $\mathbf{1 6} \mathbf{b}$ are the relatively more active cytotoxic agents against $(\mathrm{H} 460)$ tumor cells. (Curve 1 , Curve 2). 
We can assume that the kind of substituents in position 5 and 6 of the tested compounds are effective with respect to the cytotoxic activity. The presence of thionyl or ethyl carboxylate at position 5 and Hydrogen proton at position 6 increases the cytotoxic activity. In contrast, the presence of nitrile group or two carboxylate groups at positions 5 and 6 decreases the cytotoxic activity.

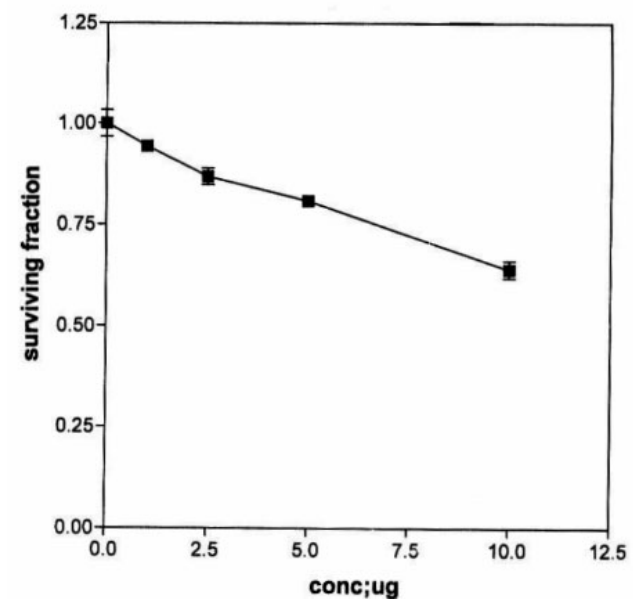

Curve 1. Cytotoxic activity of compound 8a against $\mathrm{H} 460$.

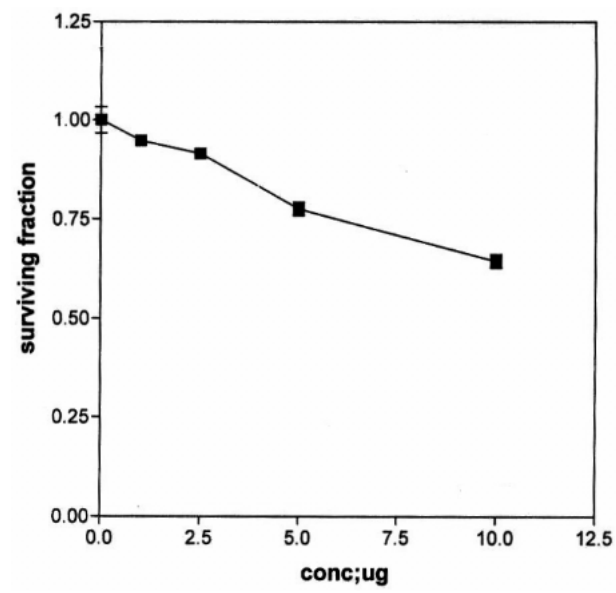

Curve 2. Cytotoxic activity of compound 16b against $\mathrm{H} 460$. 


\section{References}

[1] Gangjee A, Aldair O, Queener S F.

Pneumocystis carinii and Toxoplasma gondii dihydrofolate reductase inhibitors and antitumor agents: synthesis and biological activities of 2,4-diamino-5-methyl-6-[(monosubstituted- anilino)methyl] - pyrido[2,3- $d$ ]pyrimidines.

J. Med. Chem. 1999; 42(13): 2447-2455.

[2] Grivsky E M, Lee S; Sigel C W, Duch D S, Nichol C A.

Synthesis of antitumor activity of 2,4-diamino-6-(2,5-dimethoxybenzyl)-5-methylpyrido[2,3- $d$ ] pyrimidine.

J. Med. Chem. 1980; 23(3): 327-329.

[3] Furuya S, Ohtaki T.

Pyrido[2,3- - ] pyrimidines and their use as endothelin anatagonists.

Eur. Pat. Appl. Ep. 608565, 1994; Chem. Abstr. 1994; 121: 205395w.

[4] Heber D, Heers C, Ravens U.

Positive inotropic activity of 5-amino-6-cyano-1,3-dimethyl-1,2,3,4tetrahydropyrido[2,3-d]pyrimidine-2,4-dione in cardiac muscle from guinea pig and man. Part 6: compounds with positive inotropic activity.

Pharmazie 1993; 48(7): 537-541.

[5] Sakuma Y, Hasegawa M, Kataoka K, Hoshina K, Yamazaki N, Kadota T, Yamaguchi H.

Preparation of bicyclic pyrimidine derivatives and pharmaceutical compositions containing them for treatment of hypoxemia.

PCT Int. Appl. WO 91 05,785, 1989; Chem. Abstr. 1991; 115: $71646 \mathrm{u}$.

[6] Singh G, Singh G, Yadav A K, Mishraa K.

Synthesis and antimicrobial evaluation of some new pyrido[2,3-d] pyrimidines and their riboufuranosides.

Ind. J. Chem. 2002; 41(2): 430-432.

[7] Zakharov A V, Gavrilov M Yu, Demina L M, Novoselova G N, Gornova N A, Konshin M Ye.

Derivatives of 1-aryl-2-vinylpyrido[2,3-d]pyrimidine-4-ones: synthesis and antimicrobial activity.

Kim.-Farm. Zh. 1994; 28(11): 24-26. Chem. Abstr. 1996; 124: 29692s.

[8] DeGraw J I, Christie P H, Clowell W T, Sirotnak F M. Synthesis and antifolate properties of 5,10-ethano-5,10-dideazaaminoprotein.

J. Med. Chem. 1992; 35(2): 320-324. 
[9] Katritzky A R, Rees C W, Eds.

In: Comperhensive Heterocyclic Chemistry,

(Boulton A. J.; McKillop A., Eds.), Volume 3, Pergamon Press, Oxford, 1984: 129-261..

[10] Tamura G, Sakaguchi T, Kawasaki T, Kita Y.

A regioselective synthesis of pyrrolo- and pyrido[2,3-d]pyrimidine2,4-diones.

Heterocycles 1975; 3(2): 183-186.

[11] Quiroga J, Hormaza A, Insuasty B, Nogueras M, Sánchez A., Hanold N, Meier $\mathrm{H}$.

Synthesis of pyrido[2,3-d]pyrimidine by reaction of aminopyrimidine4-one with benzylidene meldrum's acid derivatives.

J. Heterocycl. Chem. 1997; 34: 521-524.

[12] Hirota K, Kuki H, Maki Y.

Novel synthesis of pyrido[3,4- $d$ ]pyrimidines, pyrido[2,3- $d]$ pyrimidines and quinazolines via palladium-catalyzed oxidative coupling.

Heterocycles 1994; 37(1): 563-570.

[13] Srivastava P, Saxena A S, Ram V J.

An elegant approach towards the regioselective synthesis of deazalumazines through nucleophilic induced ring transformation reactions of 6-aryl-3-cyano-4-methylthio-2H-pyran-2-one.

Synthesis 2000: 541-544.

[14] Broom A D, Shim J L, Anderson G L.

Pyrido[2,3- $d$ ]pyrimidines. IV. Synthetic studies leading to various oxopyrido[2,3- $d$ ] pyrimidines.

J. Org. Chem.1976; 41(7): 1095-1099.

[15] Devi I, Kumar B S D, Bhuyan P J.

A novel three-component one-pot synthesis of pyrano[2,3-d] pyrimidines and pyrido[2,3-d]pyrimidines using microwave heating in the solid state.

Tetrahedron Lett. 2003, 44(45): 8307-8310.

[16] Quiroga J, Insuasty H, Insuasty B, Abonia R, Cobo J, Sánchez A, Nogueras $\mathrm{M}$.

New aspects on the selective synthesis of 7-arylpyrido[2,3-d] pyrimidines.

Tetrahedron 2002; 58(24): 4873-4877.

[17] Tadros A B, Abd El Naby B A.

Marine anti-corrosion paints based on thiouracil compounds.

Anti-Corrosion Methods and Materials 2000; 47(4): 211-214.

[18] Grayer R J, Harbone J B.

A survey of antifungal compounds from higher plants.

Phytochemistry 1994; 37: 19-42. 
[19] Muanza D N, Kim B W, Euler K L, Williams L.

Antibacterial and antifungal activity of nine medical plants from Zaire.

International J. Pharmacog. 1994; 32: 337-345.

[20] Irob O N, Moo-Young M, Anderson W A.

Antimicrobial activity of Annato extract.

International J. Pharmacog. 1996; 34: 87-90.

[21] Skehan P, Storeng R.

New coloremetric cytotoxic assay for anti-cancer drug screening.

J. Natl. Cancer Inst. 1990; 82: 1107-1112.

[22] Yshihisa M, Tohru U, Michikok K, Yuzuru S, Toshitaka M.

Synthetic studies of nucleic acid-purines and related compounds.

I. Synthesis of hypoxanthine.

Yakugaku Zasshi 1957; 77: 680-688. Chem. Abstr. 1957 ;51: 16494c.

[23] Abdel-Khalik M M, Elnagdi M H, Agamy S M.

Studies with functionally substituted heteroaromatics: the chemistry of N-phenylhydrazonylalkylpyridinium salts and of phenylhydazonylalkylbenzoazoles.

Synthesis 2000; 1166-1169.

[24] Pretsch E, Simon W, Seibl J, Clerc T.

In : Tables of spectral data for determination of organic compounds.

Springer-Verlag, Berlin, 2nd Edition. 1989: 320.

[25] Rene L, Poncent J, Auzou G.

A one pot synthesis of $\beta$-cyanoenamines.

Synthesis 1986; 419-420. 